\title{
Mitochondrial complex I deficiency leads to the retardation of early embryonic development in the Ndufs 4 knockout mice
}

\author{
Mei Wang ${ }^{1,2}$, Ya-Ping Huang ${ }^{1,2}$, Han Wu ${ }^{2}$, Ke Song ${ }^{3}$, Cong Wan $^{2}$, A-Ni Chi ${ }^{2}$, Ya-Mei Xiao ${ }^{\text {Corresp.. }}{ }^{1}$, Xiao- \\ Yang Zhao ${ }^{\text {Corresp. } 2}$ \\ ${ }^{1}$ State Key Laboratory of Developmental Biology of Freshwater Fish, Hunan Normal University, Changsha, China \\ 2 Department of Developmental Biology, School of Basic Medical Sciences, Southern Medical University, Guangzhou, China \\ 3 School of Basic Medical Sciences, Southern Medical University, Guangzhou, China \\ Corresponding Authors: Ya-Mei Xiao, Xiao-Yang Zhao \\ Email address: yameix@hunnu.edu.cn, zhaoxiaoyang@smu.edu.cn
}

Background. The NDUFS4 gene encodes an 18-kD subunit of mitochondria complex I, and mutations in this gene lead to the development of a severe neurodegenerative disease called Leigh syndrome (LS) in humans. To investigate the disease phenotypes and molecular mechanisms of Leigh syndrome, the Ndufs4 knockout (KO) mouse has been widely used as a novel animal model. Because the homozygotes cannot survive beyond child-bearing age, whether Ndufs4 and mitochondrial complex I influence early embryonic development remains unknown. In our study, we attempted to investigate embryonic development in Ndufs4 KO mice, which can be regarded as a Leigh disease model and were created through the CRISPR (clustered regularly interspaced short palindromic repeat) and Cas9 (CRISPR associated)-mediated genome editing system.

Methods. We first designed a single guide RNA (sgRNA) targeting exon 2 of Ndufs 4 to delete the NDUFS4 protein in mouse embryos to mimic Leigh syndrome. Then, we described the phenotypes of our mouse model by forced swimming and the open-field test as well as by assessing other behavioral characteristics. Intracytoplasmic sperm injection (ICSI) was performed to obtain KO embryos to test the influence of NDUFS4 deletion on early embryonic development.

Results. In this study, we first generated Ndufs4 KO mice with physical and behavioral phenotypes similar to Leigh syndrome using the CRISPR/Cas9 system. The low developmental rate of KO embryos that were derived from knockout gametes indicated that the absence of NDUFS4 impaired the development of preimplantation embryos.

Discussion. In this paper, we first obtained Ndufs4 KO mice that could mimic Leigh syndrome using the CRISPR/Cas9 system. Then, we identified the role of NDUFS4 in early embryonic development, shedding light on its roles in the respiratory chain and fertility. Our model provides a useful tool with which to investigate the function of Ndufs4. Although the pathological mechanisms of the disease need to be discovered, it helps to understand the pathogenesis of NDUFS4 deficiency in mice and its effects on human diseases. 
1 Mitochondrial complex I deficiency leads to the retardation of early embryonic

2 development in $N d u f_{s} 4$ knockout mice

3

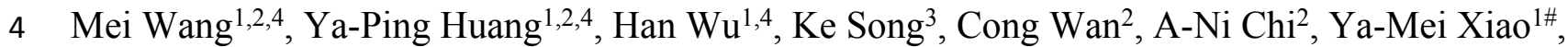
5 Xiao-Yang Zhao ${ }^{2 \#}$

6

\section{Affiliations:}

${ }^{1}$ State Key Laboratory of Developmental Biology of Freshwater Fish, Hunan Normal University, Changsha, Hunan, 410081, China

${ }^{2}$ Department of Developmental Biology, School of Basic Medical Sciences, Southern Medical University, Guangzhou, Guangdong, 510000, China

${ }^{3}$ School of Basic Medical Sciences, Southern Medical University, Guangzhou, Guangdong, 510000, China

${ }^{4}$ These authors contributed equally to this work.

\section{\#Corresponding authors: Xiao-Yang Zhao and Ya-Mei Xiao}

Address correspondence to: Xiao-Yang Zhao, 1838 North Street of Guangzhou Avenue, Baiyun District, Guangzhou, Guangdong, 510000, P R. China; Email: zhaoxiaoyang@smu.edu.cn or YaMei Xiao, 36 Lushan Road, Yuelu District, Changsha, Hunan, 410081, P R. China; Email: yameix@hunnu.edu.cn 


\section{ABSTRACT}

Background. The NDUFS4 gene encodes an 18-kD subunit of mitochondria complex I, and mutations in this gene lead to the development of a severe neurodegenerative disease called Leigh syndrome (LS) in humans. To investigate the disease phenotypes and molecular mechanisms of Leigh syndrome, the $N d u f s 4$ knockout $(\mathrm{KO})$ mouse has been widely used as a novel animal model. Because the homozygotes cannot survive beyond child-bearing age, whether $N d u f_{S} 4$ and mitochondrial complex I influence early embryonic development remains unknown. In our study, we attempted to investigate embryonic development in Ndufs $4 \mathrm{KO}$ mice, which can be regarded as a Leigh disease model and were created through the CRISPR (clustered regularly interspaced short palindromic repeat) and Cas9 (CRISPR associated)mediated genome editing system.

Methods. We first designed a single guide RNA (sgRNA) targeting exon 2 of $N d u f_{S} 4$ to delete the NDUFS4 protein in mouse embryos to mimic Leigh syndrome. Then, we described the phenotypes of our mouse model by forced swimming and the open-field test as well as by assessing other behavioral characteristics. Intracytoplasmic sperm injection (ICSI) was performed to obtain KO embryos to test the influence of NDUFS4 deletion on early embryonic development.

Results. In this study, we first generated $N d u f_{s} 4 \mathrm{KO}$ mice with physical and behavioral phenotypes similar to Leigh syndrome using the CRISPR/Cas9 system. The low developmental rate of $\mathrm{KO}$ embryos that were derived from knockout gametes indicated that the absence of NDUFS4 impaired the development of preimplantation embryos.

Discussion. In this paper, we first obtained $N d u f_{s} 4 \mathrm{KO}$ mice that could mimic Leigh syndrome using the CRISPR/Cas9 system. Then, we identified the role of NDUFS4 in early embryonic development, shedding light on its roles in the respiratory chain and fertility. Our model provides a useful tool with which to investigate the function of $N d u f s 4$. Although the pathological mechanisms of the disease need to be discovered, it helps to understand the pathogenesis of NDUFS4 deficiency in mice and its effects on human diseases.

\section{INTRODUCTION}

Mitochondria are the major energy-producing organelles in eukaryotes, and the $N d u f_{s} 4$ encodes an 18-kD subunit of mitochondrial complex I (CI), which is the largest protein assembly of the respiratory chain and forms the major entry-point of electrons into the oxidative phosphorylation system (OXPHOS). Patients with mutations in NDUFS4 will develop Leigh syndrome, a neurodegenerative disease with onset in infancy or early childhood (Anderson et al. 2008; Lamont et al. 2017), and possess a CI assembly defect along with a severe abolishment of CI 
activity (Petruzzella et al. 2001). Patients typically fail to thrive and often develop motor coordination dysfunction, episodic vomiting, blindness, hypotonia, seizures, and ataxia. Mutation of the Ndufs4 gene in mice leads to physical and behavioral symptoms, including hair loss, growth retardation, and an inactive and slow response, causing fatal encephalomyopathy, which can mimic CI disorder and cause a Leigh-like syndrome (Ingraham et al. 2009; Kruse et al. 2008). Therefore, $N d u f s 4$ deletion mice are excellent models with which to study the phenotypes and molecular mechanisms of mitochondrial complex I deficiency and Leigh syndrome.

Energy metabolism is involved in numerous biological events, including fertility and embryogenesis. Researchers found that a higher potential for continued embryogenesis and implantation in humans was associated with embryos that developed from oocytes with greater ATP content (Van Blerkom et al. 1995). Another report had shown that mitochondrial dysfunction in mouse oocytes results in preimplantation embryo arrest in vitro (Thouas et al. 2004). Moreover, many aberrations during early mouse embryonic development and the increasing health risks in the offspring may be caused by numerous dysregulated genes related to mitochondrial complex I in IVF (in vitro fertilization) embryos (Ren et al. 2015). These data indicated that both mitochondria and CI play crucial roles in embryonic development. NDUFS4, a critical subunit of CI whose effect on embryogenesis and fertility remains unknown, is worthy for further research, which could shed light on the relationship between mitochondria and early embryos.

In previous studies, $N d u f S 4 \mathrm{KO}$ mice were obtained by TALEN (Transcription activator-like effector nucleases) (Kruse et al. 2008) or homologous recombination (Ingraham et al. 2009; Leong et al. 2012). Recently, the CRISPR/Cas9 system has been considered as a revolutionary genome-editing technique (Hsu et al. 2014; Mali et al. 2013a; Segal 2013). The CRISPR/Cas9 system was first discovered as an RNA-based adaptive immune system in bacteria and archaea, which used CRISPR to identify and Cas proteins to cut exogenous DNA sequences (Cong et al. 2013; Jinek et al. 2012; Mali et al. 2013b; Wiedenheft et al. 2012). Traditionally, to generate mice with gene modification, targeted mouse embryonic stem cells (ESCs) were injected into blastocysts to obtain mice with chimeric germ cells. Here, we injected capped polyadenylated Cas9 mRNA and sgRNA into mouse zygotes to generate mutant mice in one step, which was cheaper and time-saving (Wang et al. 2013; Yang et al. 2014a; Yang et al. 2013; Zhou et al. 2014).

In this study, we first generated $N d u f s 4 \mathrm{KO}$ mice with physical and behavioral phenotypes similar to Leigh-like syndrome using the CRISPR/Cas9 system on mouse embryos. We further found that knockout of Ndufs4 in mice impaired the embryonic developmental rate during preimplantation stages. Our data revealed that NDUFS4 or mitochondria complex I plays an important role in early embryonic development.

\section{MATERIALS AND METHODS}

\section{Animal experiments}


117 B6D2F1 mice were bred with C57BL/6 female (purchased from laboratory animal center of

118

119

120

121

122

123

124

125

126

127

128

129

130

131

132

133

134

135

136

137

138

139

140

141

142

143

144

145

146

147

148

149

150

151

152

153

154

Southern Medical University) and DBA2 male (purchased from Nanjing Biomedical Research Institute of Nanjing University) mice at the laboratory animal center of Southern Medical University. ICR mice were purchased from Guangdong Medical Laboratory Animal Center. All experiments were approved by the Southern Medical University ethics committee (00125817).

RNA synthesis

To make capped polyadenylated Cas9 mRNA, px330 was used as the template DNA to amplify the Cas9 coding sequence, and the T7 promoter was added by using the primers T7-Cas9-F and Cas9-R. The PCR product was purified using the ZYMO gel recovery kit, and then, it was used as the template for in vitro transcription using the mMESSAGE mMACHINE SP6 or T3 kit (Invitrogen). For the sgRNA, the T7 promoter was added by PCR amplification using template px330 and the primers T7-sgRNA-F and sgRNA-R. Purified T7-sgRNA PCR products were used as a template for in vitro transcription using T7 RNA polymerase (New England Biolabs). After in vitro transcription, the capped polyadenylated Cas9 mRNA and sgRNA were purified with the OMEGA microelute RNA clean-up kit.

\section{Pronuclear microinjection and embryo transfer}

Pronuclear microinjection was performed based on a previously reported Nature protocol (Yang et al. 2014b). Zygotes were collected from the oviducts of hormone-superovulated B6D2F1 female mice that were crossed with B6D2F1 male mice one day before they were sacrificed. Then, a mixture of the aforementioned Cas 9 mRNA (100 ng/ $\mu \mathrm{l})$ and $\operatorname{sgRNA}(50 \mathrm{ng} / \mu \mathrm{l})$ was injected into one of the pronuclei using a micromanipulator system (Narishige) that was equipped with a microinjector (FemtoJet; Eppendorf). After nucleotide injection, we cultured the zygotes to the blastocyst stage in vitro and then transferred the embryos into the uteri of recipient pseudopregnant females. Full-term pups were delivered naturally or by cesarean section.

\section{Genotyping}

To verify the genotype, genomic DNA was extracted from the toes of the 7 10-day-old postnatal mice using the OMEGA MicroElute Genomic DNA Kit. The sequence around the target site (536 bp) was amplified using the genomic DNA template and the primers $N d u f s 4$-test-F and Ndufs4-test-R. Then, the PCR products were cloned into the TA Cloning Vector and subsequently underwent sequencing. The specific primers used in this paper are as follows: T7Cas9-F, ttaatacgactcactataggGGAGAATGGACTATAAGGACCACGAC; Cas9-R, GCGAGCTCTAGGAATTCTTAC; T7-sgRNA-F, ttaatacgactcactataggTA TAACAGTTGATGAGAAAC; sgRNA-R, AAAAGCACCGACTCGGTGCC; $N d u$ - $f s 4$-test-F, TACTGTTCAAGCAGCGTGTT; and $N d u f_{s} 4$-test-R, ATGGGCTCACATTA CCAC. 


\section{Western blot}

Livers were placed into lysis buffer (10 mM Tris- $\mathrm{HCl}[\mathrm{pH} 8.0], 10 \mathrm{mM} \mathrm{NaCl}$, and $0.5 \% \mathrm{NP}-40)$ containing protease inhibitors (Roche) and homogenized by a shaker. Then, lysates were centrifuged at $12,000 \mathrm{~g}$ for $20 \mathrm{~min}$ at $4^{\circ} \mathrm{C}$. The supernatants containing loading buffer were boiled for $5 \mathrm{~min}$. Western blotting transfers were carried out in BioRad transblot chambers. After blocking in 5\% milk in phosphate-buffered saline Triton X100 (PBST) for $1 \mathrm{~h}$, the membranes were incubated at $4^{\circ} \mathrm{C}$ overnight with anti-NDUFS4 mouse antibody (Abcam, ab87399) followed by incubation with an HRP-conjugated secondary antibody for $1 \mathrm{~h}$ at room temperature. The signals were visualized with the Super Signal West Femto reagent (Thermo scientific).

\section{Statistical analyses}

For statistical analyses, F-tests were performed to test the equality of variances in the data between WT and KO groups. Comparisons of the significant differences between the means of the WT and KO groups were performed by unpaired t-tests. Statistical significance was reached when $\mathrm{p}<0.05 .{ }^{*} \mathrm{p}<0.05,{ }^{* *} \mathrm{p}<0.01,{ }^{* * *} \mathrm{p}<0.001$.

\section{Open-field test}

Activity monitoring was performed in a square-shaped, blue open field measuring $75 \mathrm{~cm} \times 75$ $\mathrm{cm}$ that was evenly illuminated at $15 \mathrm{lux}^{50}$. Mice were placed facing the center of the walls and were allowed to move in the apparatus; they were monitored for $10 \mathrm{~min}$ by a video camera. The resulting data were analyzed using the image processing system TopScan. The following parameters were assessed: total distance moved, velocity, mean distance to the (nearest) wall and time in the center.

\section{Intracytoplasmic sperm injection}

ICSI was performed as previously described (Zhou et al. 2016). We broke the tail of the sperms isolated from the cauda epididymis using an ultrasonic cell disruptor, and superovulated MII oocytes were collected as described above. The sperms were exposed to $5 \mathrm{mg} / \mathrm{ml}$ cytochalasin B in M2 medium, and individual sperm was injected into mature oocytes with a Piezo-driven pipette followed by culture in KOSM medium to the blastocyst stage.

\section{RESULTS}

Generation of the $N d u f_{S} 4 \mathrm{KO}$ mouse model by CRISPR

The NDUFS4 protein includes two parts, the transit peptide and the NDUFS4 mature protein. NDUFS4 is encoded by five exons, among which exon 2 encodes the first 17 amino acids of the 
190

191

192

193

194

195

196

197

198

199

200

201

202

203

204

205

206

207

208

209

210

211

212

213

214

215

216

217

218

219

220

221

222

223

224

225

226

227

228

NDUFS4 protein and the last part of the transit peptide, which is important for targeting on mitochondria (Breuer et al. 2013). To generate Ndufs 4 KO mice by CRISPR (Fig. 1A), we designed the sgRNA to target exon 2 of the $N d u f s 4$ gene (Fig. 1B). Then, we synthesized capped polyadenylated Cas9 mRNA and sgRNA by in vitro transcription and co-injected them into 89 mouse embryos at the pronuclear (PN) stage (Fig. 1A). The embryos were cultured to the blastocyst stage and transplanted into pseudopregnant female mice. Finally, we got 7 homozygous $N d u f_{S} 4 \mathrm{KO}$ pups (Fig. 1C). In 20 predicted off-target sites, we did not observe any sense mutations on the coding sequences (Supplementary Table 1, 2) (Stemmer et al. 2015; Yang et al. 2013; Zong et al. 2017). Through sequencing, we found deletions in exon 2, which hindered the synthesis of mature NDUFS4. Previous research showed that transposable element insertion into exon 3 could thoroughly diminish NDUFS4 expression (Leong et al. 2012). In our data, NDUFS4 proteins were completely abolished in these KO mice, as shown in Fig. 1D.

\section{Phenotypes of $N d u f_{s} 4$ knockout mice}

By 3 weeks after birth, all homozygous $\mathrm{KO}$ mice had begun to lose their body hair (Fig. 2A, left). However, their hair grew back during the next hair-growth cycle (Fig. 2A, right). Also, the body weight of KO mice was significantly lower than that of wild-type (WT) mice at 8 weeks both in females $(n=3, p=0.0082)$ and in males $(n=4, p=0.00142)$ (Fig. 2B).

To confirm the phenotype of the KO mice, the forced swim test was performed to estimate the motor ability of the Ndufs $4 \mathrm{KO}$ mice. In the forced swim test, WT mice could swim for nearly 20 min, while KO mice sank to the bottom as soon as they got into the water. It seemed that the Ndufs 4 KO mice were extremely frail.

Then, we performed the open-field test to record the total distance traveled and the velocity in the trial area. The open-field test is currently one of the most popular procedures in animal psychology. In fact, it has become a convenient way to measure the activity of animal models (Prut \& Belzung 2003). Motion tracker data showed that the movement tracks of 7 KO mice (Fig. $2 \mathrm{C}$, left) were much less than that of the 14 control mice (Fig. 2C, right). Locomotor disturbances with regard to the distance traveled $(\mathrm{p}<0.0001)$ and the velocity $(\mathrm{p}<0.0001)$ among Ndufs 4 knockout mice were observed compared to control mice in the open field (Fig. 2D), indicating that $N d u f_{s} 4 \mathrm{KO}$ mice suffered from the rapid deterioration of motor ability. Altogether, we verified that the $N d u f_{S} 4 \mathrm{KO}$ mice generated using the CRISPR/Cas9 system could mimic Leigh syndrome and function as a disease model.

\section{NDUFS4 affected early embryonic development in mice}

The KO mice became weak and died approximately 6 weeks after birth, and few of them survived beyond 9 weeks, which meant that the KO mice rarely generated offspring during their lifetime. Meanwhile, the KO mice could not produce pups by natural mating with WT mice. To assess the effect of NDUFS4 on embryos, we performed ICSI using KO gametes from mouse \#1 (female) and mouse \#2 (male). The KO and WT gametes were divided into four groups, and we 
229

230

231

232

233

234

235

236

237

238

239

240

241

242

243

244

245

246

247

248

249

250

251

252

253

254

255

256

257

258

259

260

261

262

263

264

265

266

267

268

performed ICSI on nearly 40 embryos in each group (Table 1). After the injection, pronuclei could be observed in each group, which indicated the fertility of Ndufs $4 \mathrm{KO}$ gametes. Subsequently, the embryos progressed from the 2-cell stage to the blastocyst stage in vitro. We did not observe significant differences in the developmental rates between embryos generated from KO sperms that were injected into WT oocytes or WT sperms that were injected into KO oocytes compared to the embryos generated from WT sperms that were injected into WT oocytes (Fig. 3A). However, the developmental rate of zygotes derived from $\mathrm{KO}$ sperms and $\mathrm{KO}$ oocytes was significantly lower than that of WT embryos at the 2-cell stage (78.4\% versus $97.5 \%)$, 4-cell stage $(62.2 \%$ versus $92.5 \%)$, morula stage $(51.4 \%$ versus $85 \%)$ and blastocyst stage $(29.7 \%$ versus $70 \%$ ). The ICSI data demonstrated that Ndufs 4 knockout impaired the preimplantation embryonic developmental ability in mice. The transplantation of $11 \mathrm{KO}$ blastocysts into pseudopregnant mice did not result in any live births. However, 7 offspring were obtained from the transplantation of 28 control blastocysts. On one hand, the transplantation data may be a result of the limited KO embryos, but on the other hand, it suggested that the $N d u f_{s} 4$ knockout blastocysts generated from KO gametes may have an extremely low efficiency to develop to fullterm.

These results raised the possibility that abnormalities in the gonads of $\mathrm{KO}$ mice may exist. To verify this hypothesis, we checked the testes and ovaries of $\mathrm{KO}$ mice. Compared with controls, the testes and ovaries of 8-week-old postnatal $\mathrm{KO}$ mice were similar in size (Fig. 3B). In addition, hematoxylin and eosin (H\&E) staining of the ovaries at 8 weeks showed that the KO ovaries harbored plenty of follicles at different stages and that the number of follicles in KO ovaries (79 and 76 oocytes in $2 \mathrm{KO}$ mice) was much greater than that in the WT ovaries (36 and 45 oocytes in 2 WT mice). It was consistent with our findings that the MII oocytes obtained from mouse \#1 ( 80 oocytes) were much more abundant than the oocytes that were obtained from a WT female mouse (40 50 oocytes) after superovulation. This result indicated that there was a spontaneous ovulation arrest in KO female mice. However, the details of this mechanism are not clear. The $\mathrm{KO}$ testes contained intact seminiferous epithelium and mature elongated spermatozoa in the seminiferous tubes (Fig. 3C).

Taken together, these data demonstrated that NDUFS4 plays a role in early embryonic development and spontaneous ovulation in mice.

\section{DICUSSION}

Leigh syndrome is a progressive neurodegenerative disorder that is caused by mitochondrial oxidative phosphorylation defects. The cause of the syndrome is complex, including mitochondrial DNA point mutations and some respiratory chain enzyme defects, such as complex I, IV and pyruvate dehydrogenase. In addition, complex I deficiency is more common than previously recognized (Lamont et al. 2017; Rahman et al. 1996). Though Leigh syndrome is a genetically heterogeneous entity (Gerards 2014), all of the biochemical defects described to date in patients with LS affect terminal oxidative metabolism and are likely to impair energy 
269

270

271

272

273

274

275

276

277

278

279

280

281

282

283

284

285

286

287

288

289

290

291

292

293

294

295

296

297

298

299

300

301

302

303

304

305

306

307

308

production. Typically, OXPHOS dysfunction mostly affects cell types that heavily rely on mitochondrial ATP generation.

Preimplantation mouse embryonic development includes the processes of zygote cleavage to blastocysts, especially from the morulae stage to the blastocyst stage, which involves a series of high energy consumption events. Previous reports have suggested that low ATP was not only associated with mouse MII oocyte spindle impairment (Zhang et al. 2006) but also associated with a reduction in the quality of the developed embryos (Van Blerkom 2004). In our study, the KO zygotes had a worse embryonic cleavage rate than that of heterozygous and WT embryos. It indicated that the low developmental rate may be due to $N d u f_{s} 4$ knockout. Consequently, there may be some correlation of low ATP and early embryos retardation with the knockout of Ndufs 4 . However, we could generate homozygous mutant mice by Cas9 and sgRNA mRNA injection into WT zygotes at a relatively normal frequency, which may have been due to the normal NDUFS4 protein sustained from WT zygotes.

In 2013, Federica Valsecchi et al found that the primary fibroblasts of $\mathrm{Ndufs}_{\mathrm{S}^{-/}}$mice displayed increased ROS levels and aberrant mitochondrial morphology (Valsecchi et al. 2013). ROS cannot only alter most types of cellular molecules but also induce developmental block and retardation (Guerin et al. 2001). However, whether the ROS accumulation and aberrant mitochondrial morphology in Ndufs $4 \mathrm{KO}$ embryos led to retardation remains to be determined.

In mammals, CI requires the correct assembly of 45 subunits encoded by both nuclear and mitochondrial DNA in order to function correctly (Koopman et al. 2010), and the structure is evolutionary conserved (Leong et al. 2012). Because of the complexity of CI, the deletion of the other units of CI may also lead to the same result as Ndufs $4 \mathrm{KO}$ or influence the function of CI. Transcriptome profile data showed that there were many mitochondria-related genes that were differentially expressed between in vitro fertilization (IVF) and in vivo fertilization (IVO) embryos, including NDUFS1, NDUFV1, NDUFA2, NDUFB8 and NDUFA9, which are subunits of CI together with NDUFS4 (Ren et al. 2015). The abnormal expression of these genes led to dysfunction of the mitochondria and, subsequently, IVF-induced embryonic defects. Notwithstanding, the role of NDUFS4 and other CI subunits in embryonic development also needs to be further investigated.

In addition, mutations of other genes encoding enzymes related to the respiratory chain lead to early embryonic death. Surfl encodes one of the assembly proteins involved in the formation of cytochrome c oxidase (COX); mutations in this gene also contributed to the phenotype of Leighlike syndrome, and constitutive knockout of Surfl was associated with increased embryonic lethality (Agostino et al. 2003). Knockout of Slc25a19 caused mitochondrial thiamine pyrophosphate depletion and embryonic lethality. Although the reason behind embryonic retardation is unknown, these data may provide ideas for us to investigate the mechanisms in Ndufs $4 \mathrm{KO}$ embryos.

\section{CONCLUSIONS}

In summary, $N d u f_{S} 4 \mathrm{KO}$ mice were first obtained using the CRISPR-Cas9 system, which is a 
309

310

311

312

313

314

315

316

317

318

319

320

321

322

323

324

325

326

327

328

329

330

331

332

333

334

335

336

337

338

339

340

341

342

343

344

345

346

347

more efficient and time-saving option for generating genetically modified animals than that used in previous studies. We not only observed hair loss and weight loss but also motor impairment in Ndufs 4 KO mice. A role for NDUFS4 in early embryonic development and ovulation was indicated, shedding light on its roles in the respiratory chain and fertility. Moreover, our model provided a useful tool with which to investigate the function of $N d u f_{s} 4$, thus helping to understand the pathogenesis of NDUFS4 deficiency.

\section{ACKNOWLEDGMENTS}

We thank Zhao-Ting Liu, Yu-Jia Shi and Jing Wang for reviewing the manuscript.

\section{REFERENCES}

Agostino A, Invernizzi F, Tiveron C, Fagiolari G, Prelle A, Lamantea E, Giavazzi A, Battaglia G, Tatangelo L, Tiranti V, and Zeviani M. 2003. Constitutive knockout of Surf1 is associated with high embryonic lethality, mitochondrial disease and cytochrome c oxidase deficiency in mice. Human Molecular Genetics 12:399-413. $10.1093 / \mathrm{hmg} / \mathrm{ddg} 038$

Anderson SL, Chung WK, Frezzo J, Papp JC, Ekstein J, DiMauro S, and Rubin BY. 2008. A novel mutation in NDUFS4 causes Leigh syndrome in an Ashkenazi Jewish family. J Inherit Metab Dis 31 Suppl 2:S461-467. 10.1007/s10545-008-1049-9

Breuer ME, Willems PH, Smeitink JA, Koopman WJ, and Nooteboom M. 2013. Cellular and animal models for mitochondrial complex I deficiency: a focus on the NDUFS4 subunit. IUBMB Life 65:202-208. 10.1002/iub.1127

Cong L, Ran FA, Cox D, Lin S, Barretto R, Habib N, Hsu PD, Wu X, Jiang W, Marraffini LA, and Zhang F. 2013. Multiplex genome engineering using CRISPR/Cas systems. Science 339:819-823. 10.1126/science.1231143

Gerards M. 2014. Leigh syndrome: the genetic heterogeneity story continues. Brain 137:2872-2873. 10.1093/brain/awu264

Guerin P, El Mouatassim S, and Menezo Y. 2001. Oxidative stress and protection against reactive oxygen species in the pre-implantation embryo and its surroundings. Hum Reprod Update 7:175-189.

Hsu PD, Lander ES, and Zhang F. 2014. Development and applications of CRISPR-Cas9 for genome engineering. Cell 157:1262-1278. 10.1016/j.cell.2014.05.010

Ingraham CA, Burwell LS, Skalska J, Brookes PS, Howell RL, Sheu SS, and Pinkert CA. 2009. NDUFS4: creation of a mouse model mimicking a Complex I disorder. Mitochondrion 9:204-210. 10.1016/j.mito.2009.02.001

Jinek M, Chylinski K, Fonfara I, Hauer M, Doudna JA, and Charpentier E. 2012. A programmable dual-RNA-guided DNA endonuclease in adaptive bacterial immunity. Science 337:816.

Koopman WJ, Nijtmans LG, Dieteren CE, Roestenberg P, Valsecchi F, Smeitink JA, and Willems PH. 2010. Mammalian mitochondrial complex I: biogenesis, regulation, and reactive oxygen species generation. Antioxid Redox Signal 12:1431-1470. 10.1089/ars.2009.2743

Kruse SE, Watt WC, Marcinek DJ, Kapur RP, Schenkman KA, and Palmiter RD. 2008. Mice with mitochondrial complex I deficiency develop a fatal encephalomyopathy. Cell Metab 7:312-320. 10.1016/j.cmet.2008.02.004

Lamont RE, Beaulieu CL, Bernier FP, Sparkes R, Innes AM, Jackel-Cram C, Ober C, Parboosingh JS, and Lemire EG. 
2017. A novel NDUFS4 frameshift mutation causes Leigh disease in the Hutterite population. Am J Med Genet A 173:596-600. 10.1002/ajmg.a.37983

Leong DW, Komen JC, Hewitt CA, Arnaud E, McKenzie M, Phipson B, Bahlo M, Laskowski A, Kinkel SA, Davey GM, Heath WR, Voss AK, Zahedi RP, Pitt JJ, Chrast R, Sickmann A, Ryan MT, Smyth GK, Thorburn DR, and Scott HS. 2012. Proteomic and metabolomic analyses of mitochondrial complex I-deficient mouse model generated by spontaneous B2 short interspersed nuclear element (SINE) insertion into NADH dehydrogenase (ubiquinone) Fe-S protein 4 (Ndufs4) gene. J Biol Chem 287:20652-20663. 10.1074/jbc.M111.327601

Mali P, Esvelt KM, and Church GM. 2013a. Cas9 as a versatile tool for engineering biology. Nat Methods 10:957963. 10.1038/nmeth.2649

Mali P, Yang L, Esvelt KM, Aach J, Guell M, DiCarlo JE, Norville JE, and Church GM. 2013b. RNA-guided human genome engineering via Cas9. Science 339:823-826. 10.1126/science.1232033

Petruzzella V, Vergari R, Puzziferri I, Boffoli D, Lamantea E, Zeviani M, and Papa S. 2001. A nonsense mutation in the NDUFS4 gene encoding the $18 \mathrm{kDa}$ (AQDQ) subunit of complex I abolishes assembly and activity of the complex in a patient with Leigh-like syndrome. Hum Mol Genet 10:529-535.

Prut L, and Belzung C. 2003. The open field as a paradigm to measure the effects of drugs on anxiety-like behaviors: a review. Eur J Pharmacol 463:3-33.

Rahman S, Blok RB, Dahl HH, Danks DM, Kirby DM, Chow CW, Christodoulou J, and Thorburn DR. 1996. Leigh syndrome: clinical features and biochemical and DNA abnormalities. Ann Neurol 39:343-351. 10.1002/ana.410390311

Ren L, Wang Z, An L, Zhang Z, Tan K, Miao K, Tao L, Cheng L, Zhang Z, Yang M, Wu Z, and Tian J. 2015. Dynamic comparisons of high-resolution expression profiles highlighting mitochondria-related genes between in vivo and in vitro fertilized early mouse embryos. Hum Reprod 30:2892-2911. 10.1093/humrep/dev228

Segal DJ. 2013. Bacteria herald a new era of gene editing. Elife 2:e00563. 10.7554/eLife.00563

Stemmer M, Thumberger T, Del Sol Keyer M, Wittbrodt J, and Mateo JL. 2015. CCTop: An Intuitive, Flexible and Reliable CRISPR/Cas9 Target Prediction Tool. PLoS ONE 10:e0124633. 10.1371/journal.pone.0124633

Thouas GA, Trounson AO, Wolvetang EJ, and Jones GM. 2004. Mitochondrial dysfunction in mouse oocytes results in preimplantation embryo arrest in vitro. Biol Reprod 71:1936-1942. 10.1095/biolreprod.104.033589

Valsecchi F, Grefte S, Roestenberg P, Joosten-Wagenaars J, Smeitink JA, Willems PH, and Koopman WJ. 2013. Primary fibroblasts of NDUFS4(-/-) mice display increased ROS levels and aberrant mitochondrial morphology. Mitochondrion 13:436-443. 10.1016/j.mito.2012.12.001

Van Blerkom J. 2004. Mitochondria in human oogenesis and preimplantation embryogenesis: engines of metabolism, ionic regulation and developmental competence. Reproduction 128:269-280. 10.1530/rep.1.00240

Van Blerkom J, Davis PW, and Lee J. 1995. ATP content of human oocytes and developmental potential and outcome after in-vitro fertilization and embryo transfer. Hum Reprod 10:415-424.

Wang H, Yang H, Shivalila CS, Dawlaty MM, Cheng AW, Zhang F, and Jaenisch R. 2013. One-step generation of mice carrying mutations in multiple genes by CRISPR/Cas-mediated genome engineering. Cell 153:910-918. 10.1016/j.cell.2013.04.025

Wiedenheft B, Sternberg SH, and Doudna JA. 2012. RNA-guided genetic silencing systems in bacteria and archaea. Nature 482:331-338. 10.1038/nature10886

Yang H, Wang H, and Jaenisch R. 2014a. Generating genetically modified mice using CRISPR/Cas-mediated genome 
406

engineering. Nat Protoc 9:1956.

Yang $\mathrm{H}$, Wang H, and Jaenisch R. 2014b. Generating genetically modified mice using CRISPR/Cas-mediated genome engineering. Nat Protoc 9:1956-1968. 10.1038/nprot.2014.134

Yang H, Wang H, Shivalila CS, Cheng AW, Shi L, and Jaenisch R. 2013. One-step generation of mice carrying reporter and conditional alleles by CRISPR/Cas-mediated genome engineering. cell 154:1370-1379. 10.1016/j.cell.2013.08.022

Zhang X, Wu XQ, Lu S, Guo YL, and Ma X. 2006. Deficit of mitochondria-derived ATP during oxidative stress impairs mouse MII oocyte spindles. Cell Res 16:841-850. 10.1038/sj.cr.7310095

Zhou J, Shen B, Zhang W, Wang J, Yang J, Chen L, Zhang N, Zhu K, Xu J, Hu B, Leng Q, and Huang X. 2014. One-step generation of different immunodeficient mice with multiple gene modifications by CRISPR/Cas9 mediated genome engineering. Int J Biochem Cell Biol 46:49-55. 10.1016/j.biocel.2013.10.010

Zhou Q, Wang M, Yuan Y, Wang X, Fu R, Wan H, Xie M, Liu M, Guo X, Zheng Y, Feng G, Shi Q, Zhao XY, Sha J, and Zhou Q. 2016. Complete Meiosis from Embryonic Stem Cell-Derived Germ Cells In Vitro. Cell Stem Cell 18:330340. 10.1016/j.stem.2016.01.017

Zong Y, Wang Y, Li C, Zhang R, Chen K, Ran Y, Qiu JL, Wang D, and Gao C. 2017. Precise base editing in rice, wheat and maize with a Cas9- cytidine deaminase fusion. Nat Biotechnol. 10.1038/nbt.3811 


\section{Figure 1}

\section{Generation of Ndufs4 KO mouse model}

(A) Strategy for generating the Ndufs4 KO mouse model, and the procedure of our study.

(B) Schematic of the Cas9/sgRNA-targeting sites in the Ndufs4 genomic locus. The guide RNA sequence is marked by a blue line, and the protospacer-adjacent motif (PAM) sequence is labeled in rose red. (C) The NDUFS4 protein is truncated in Ndufs $4^{-/}$mice, and the right panel shows the corresponding sequence.

(D) Western blot experiments showed that the NDUFS4 protein was completely abolished in the Ndufs4 ${ }^{\%}$ mice. 
A
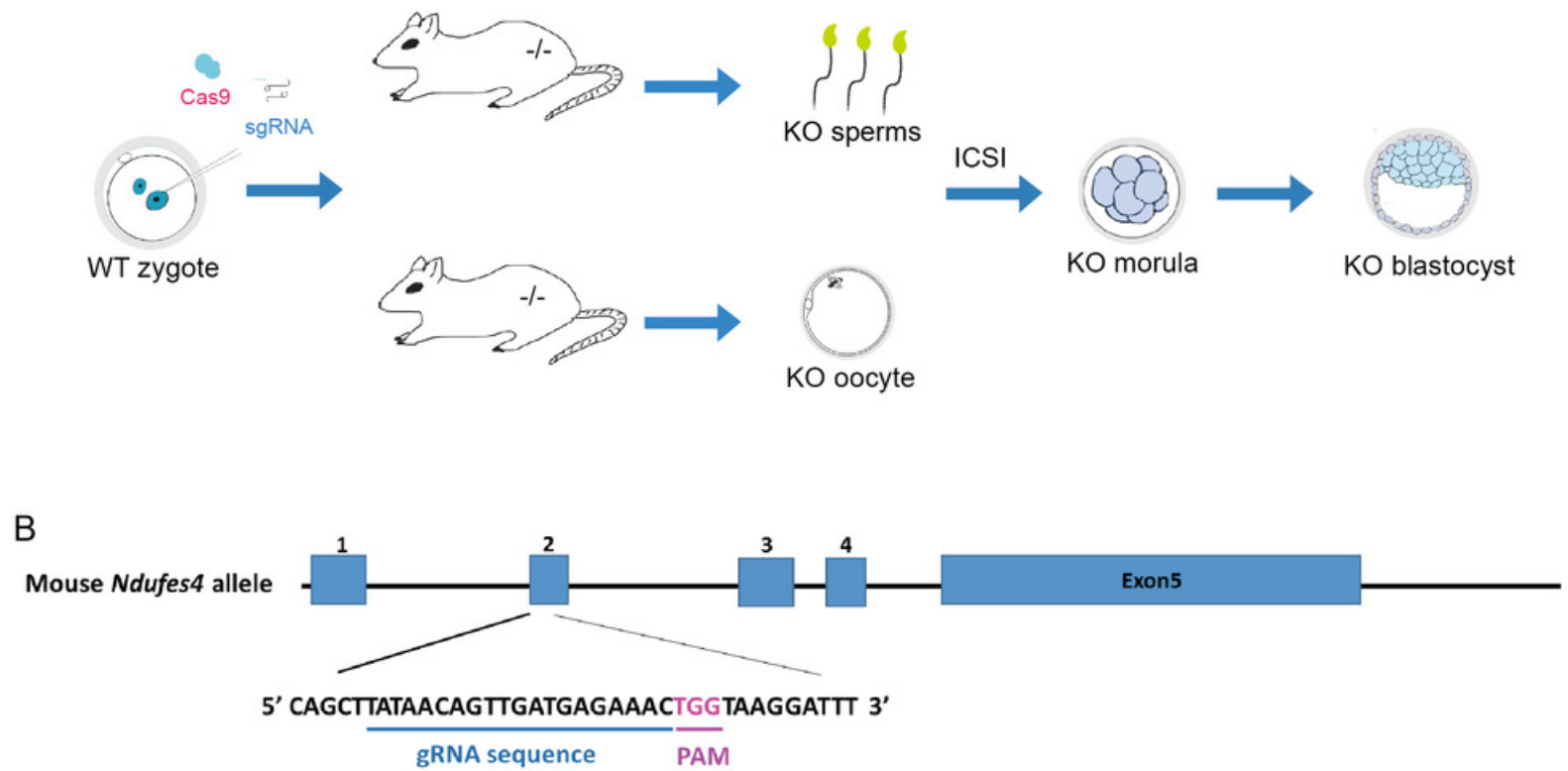

C

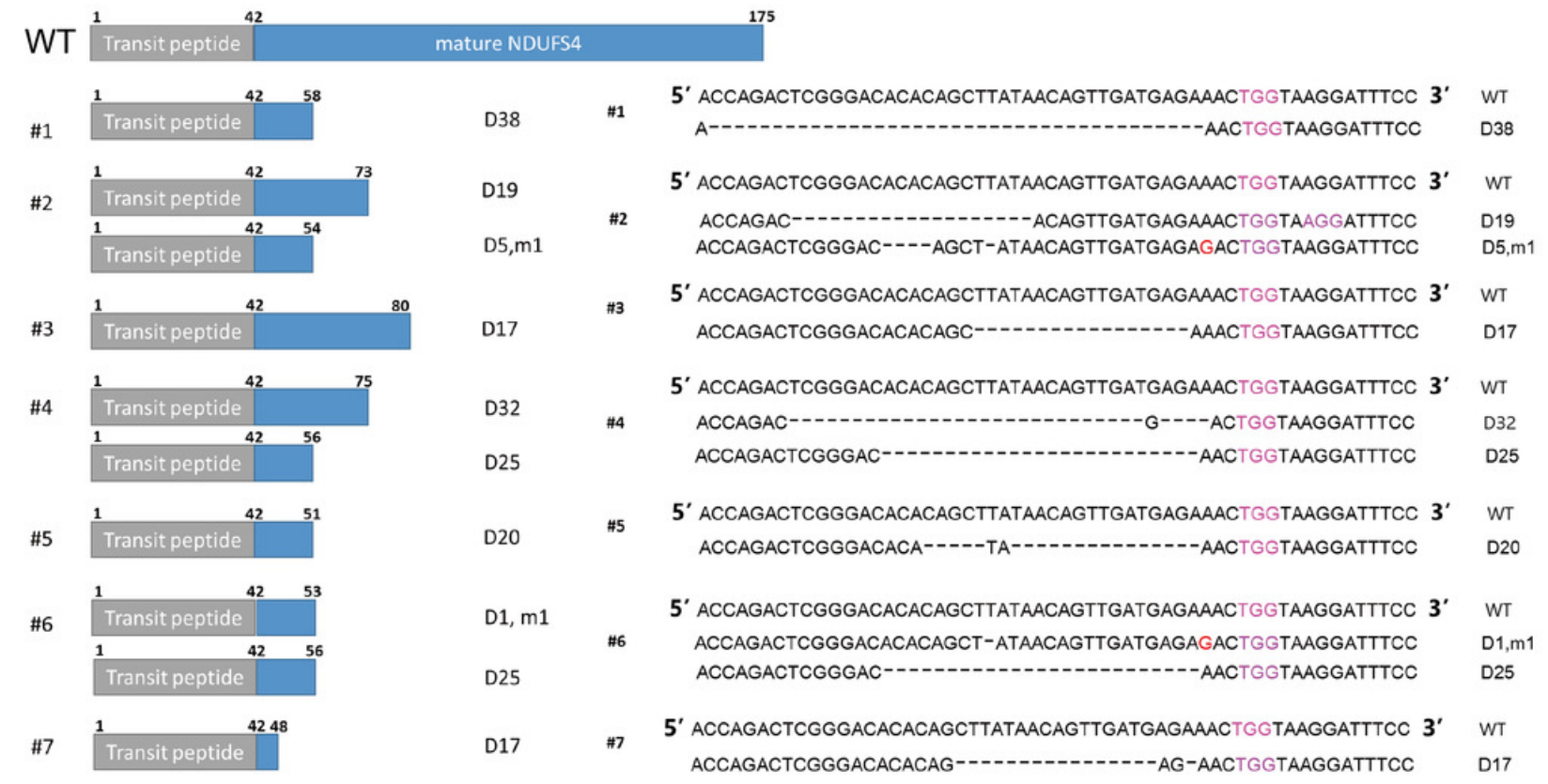

D

$42 \mathrm{kD}$

WT $\quad \# 1 \quad \# 2 \quad \# 3 \quad \# 4 \quad \# 5 \quad \# 6 \quad \# 7$

$18 \mathrm{kD}$

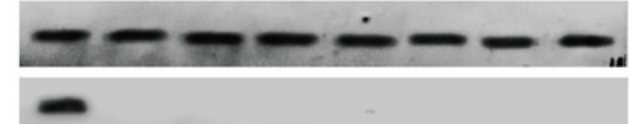

ACTIN

NDUFS4 


\section{Figure 2}

Phenotype of Ndufs4 KO mice.

(A) Ndufs4 KO mice lose their body hair at approximately 3 weeks old (left), and the characteristic would diminish along with growth (right).

(B) Both male (right) and female (left) Ndufs4 KO mice significantly lose weight at 8 weeks compared to WT mice . ${ }^{*} p<0.05$ and ${ }^{* *} p<0.01$.

(C) The motion tracker picture showing the spatial pattern of Ndufs4 KO mice (left) and WT (right) mice in the open field; KO mice walked significantly less than WT mice in the field.

(D) Ndufs4 KO mice suffered from the rapid deterioration of motor ability in the open field. KO mice moved significantly slower and less than the WT mice in the open-field test. ${ }^{* * *} p<0.001$.

A

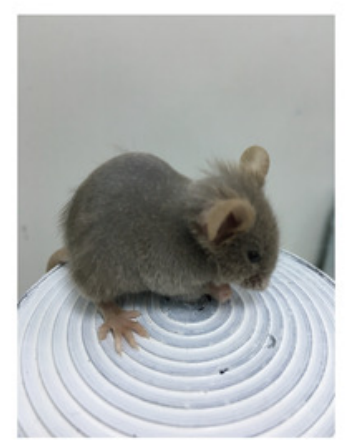

E

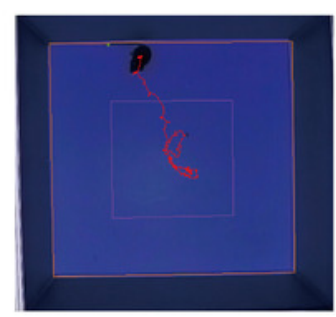

B

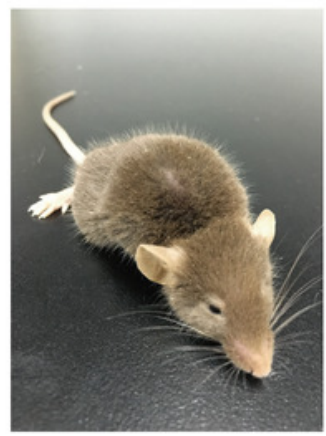

F

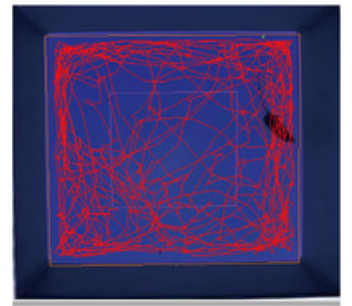

C

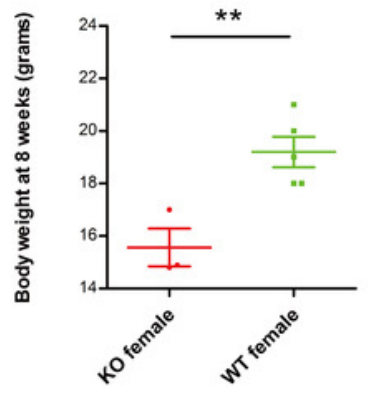

G

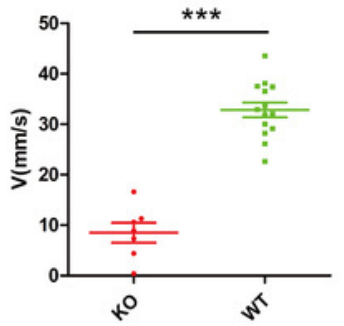

D

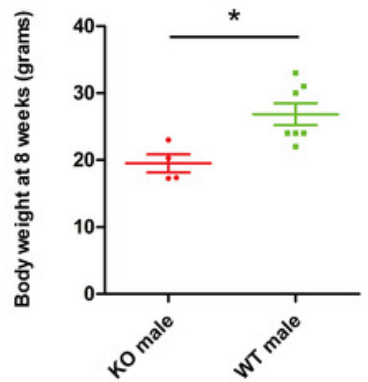

H

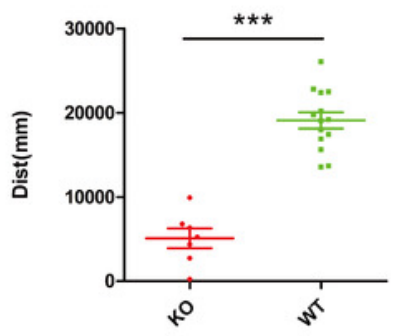




\section{Figure 3}

NDUFS4 affected the early embryos development in mice.

(A) Development of ICSI embryos from Ndufs4 KO mice in the broken line graph that refers to Table 1.

(B) The testes and ovaries of WT and Ndufs4 KO mice.

(C) HE staining of the ovaries and testes in KO and WT mice at 8 weeks old. The KO ovary contained plenty of follicles, which were more abundant than that in the WT ovary . Intact seminiferous epithelium and many mature elongated spermatozoa could be found in the KO seminiferous tubes versus WT testis. Scale bar, 50 $\mu \mathrm{m}$.

A
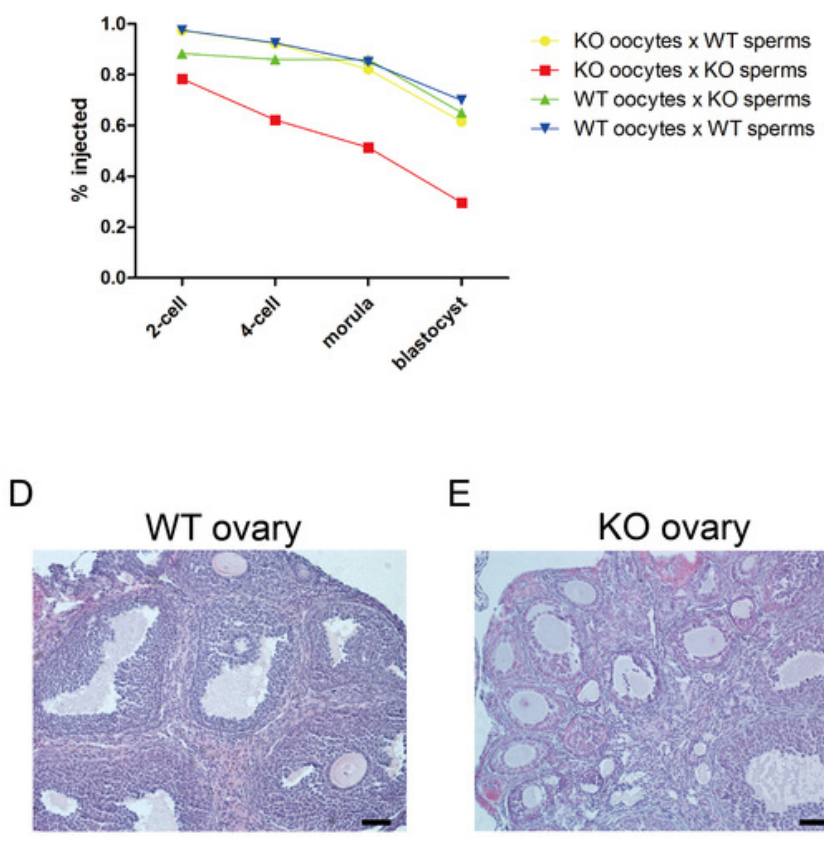

B

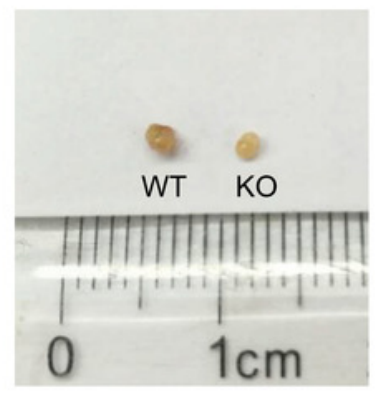

$\mathrm{E}$

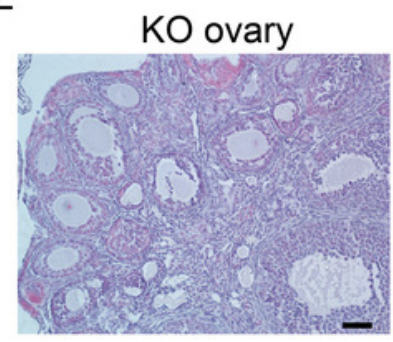

$\mathrm{F}$
C
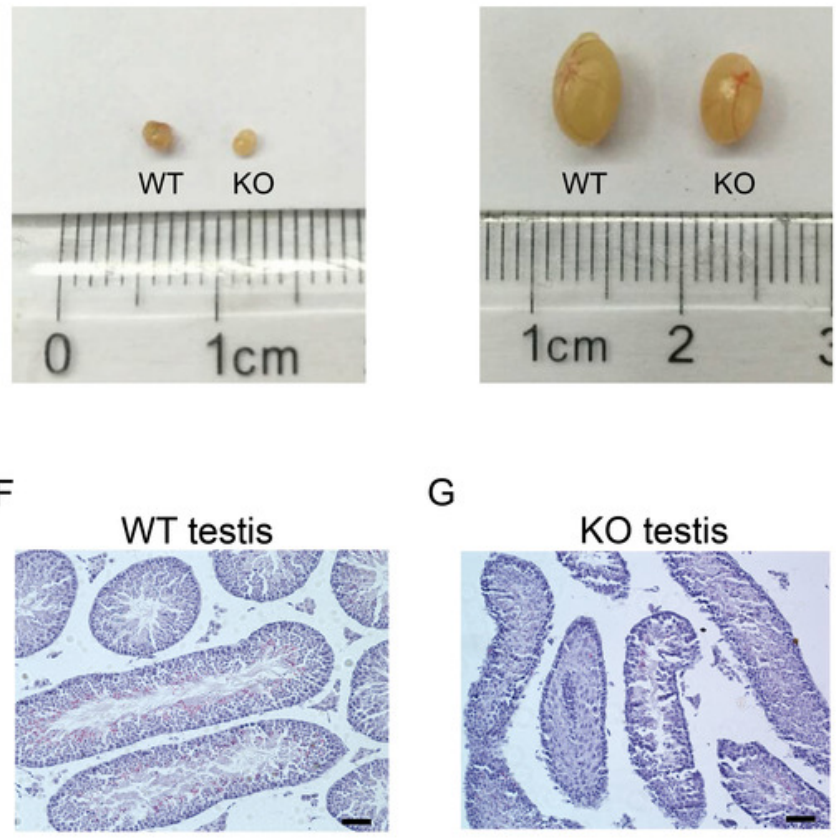

G

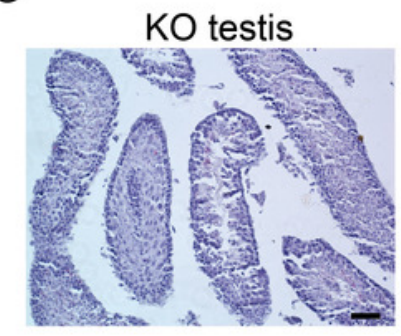




\section{Table 1 (on next page)}

Development of ICSI embryos from Ndufs4 KO mice. 
1 Table 1 Development of ICSI embryos from $N d u f_{s} 4 \mathrm{KO}$ mice

\begin{tabular}{cccccc} 
Background & $\begin{array}{c}\text { No. of } \\
\text { injected } \\
\text { oocytes }\end{array}$ & $\begin{array}{c}\text { No. of 2-cell } \\
\text { (\% injected) }\end{array}$ & $\begin{array}{c}\text { No. of 4-cell } \\
(\% \text { injected })\end{array}$ & $\begin{array}{c}\text { No. of morula } \\
(\% \text { injected })\end{array}$ & $\begin{array}{c}\text { No. of blastocyst } \\
\text { (\% injected })\end{array}$ \\
\hline $\begin{array}{c}\text { KO oocytes x WT } \\
\text { sperms }\end{array}$ & 39 & $38(97.4)$ & $36(92.3)$ & $32(82.1)$ & $24(61.5)$ \\
$\begin{array}{c}\text { KO oocytes x KO } \\
\text { sperms }\end{array}$ & 37 & $29(78.4)$ & $23(62.2)$ & $19(51.4)$ & $11(29.7)$ \\
$\begin{array}{c}\text { WT oocytes x KO } \\
\text { sperms }\end{array}$ & 43 & $38(88.4)$ & $37(86.0)$ & $37(86)$ & $28(65.1)$ \\
$\begin{array}{c}\text { WT oocytes x WT } \\
\text { sperms }\end{array}$ & 40 & $39(97.5)$ & $37(92.5)$ & $34(85)$ & $28(70)$ \\
\hline
\end{tabular}

2 